\title{
From biotechnology to nanotechnology: Philippine context
}

\author{
Marciana Agnes G. Ponsaran \\ Research Center for the Natural and Applied Sciences \\ University of Santo Tomas, 1015 Manila, Philippines
}

\begin{abstract}
In recent years, a considerable number of studies on risk assessment and regulation of nanotechnology has been published. Given the variables of risk, uncertainty and complexity of this new technology, how prepared is the Philippines to meet the challenges of nanotechnology? Nanotechnology as a fledging technology could draw wisdom from our local experiences with biotechnology.

This study examines biotechnology regulations in the Philippines in order to determine what mechanisms have been established to ward off potential harms and dangers that may arise from this technology. It investigates whether these mechanisms are adequate to address nanotechnology concerns such as health, safety, risk and regulation issues. The study makes use of the foundational principles of oversight of nanotechnologies and nanomaterials such as precaution, environmental sustainability, and inclusion of broader societal impacts, among others as reference. It seeks to leverage our experiences with biotechnology to regulate nanotechnology development in the country.
\end{abstract}

Keywords: biotechnology, nanotechnology, regulation of technologies, ethical principles

\section{INTRODUCTION}

The risk, uncertainty and complexity factors involved in nanotechnology pose a big challenge to our capability to make good decisions for the future. Decision-making under risk entails knowledge of the possible outcomes and their probabilities while decision making under uncertainty involve the lack of knowledge of the probabilities or probabilities known with insufficient precision [1].

*To whom correspondence should be addressed maponsaran@ust.edu.ph
Complexity refers to a condition somewhere between order and disorder, in which multiple parts are simultaneously behaving independently and in ways that are interconnected and dependent [2]. There have been a considerable number of studies that aims to address the problems related to risk and safety issues and regulatory challenges of nanotechnology [3-6].

As regards the concept of risk, two pertinent reports can be cited here: the Scientific Committee on Emerging and Newly Identified Health Risk (SCENIHR) ${ }^{1}$ Report and the White Paper on Nanotechnology Governance 
published by the International Risk Governance Council (IRCG) ${ }^{2}$. Both groups emphasize the lack of scientific data on the possible risks of nanoparticles with reference to human health and environment. Both acknowledged the relevance of a paradigm of risk management and communication. Both stressed the need to fill in the information gaps required for risk assessment of nanotechnology products. Both supported the need for an international framework with which to address risks issues at a global level.

The Philippines recognizes the potential for nanotechnology in addressing its developmental needs by leveraging on indigenous resources from its diverse marine, forest, and agricultural sectors [7]. Considering that the Philippines had launched its National Nanotechnology Roadmap only in 2009 , there are no available mechanisms of control or regulatory standards that are nanospecific. This does not suggest that the country does not have existing guidelines on dealing with new technologies. As early as the 90's, the Philippine government had initiated a biotechnology regulatory framework. In the years that followed, specific policies and guidelines on biotechnology will be issued

${ }^{1}$ The Committee provides opinions on emerging or newly identified health and environmental risks, and on broad, complex and multidisciplinary issues requiring a comprehensive assessment of risks to consumer safety or public health and related issues not covered by other risk assessment bodies. See http://ec.europa.eu/health/ scientific_committees/-emerging/-index_en.htm, accessed on September 1, 2010.

${ }^{2}$ IRCG is a private, independent, not-for-profit foundation based in Geneva, Switzerland whose mission is to support governments, industry, NGOs and other organizations in their efforts to understand and deal with major and global risks facing society and to foster public confidence in risk governance. See White Paper on Nanotechnology: Risk Governance, International Risk Governing Council, Geneva, June 2006, Accessed at http://www.irgc.org/IMG/pdf/IRGC-_white_paper _2_PDF_final_version-2.pdf on Aug 12, 2010 through a series of Executive and Administrative Orders. This study will examine biotechnology regulations in the Philippines in order to determine if the country has adequate mechanisms which it can leverage in order address the problems of safety, risk and regulation issues in nanotechnology, using international standards and commonly accepted ethical principles.

\section{Regulating Biotechnology in the PhILIPPINES $^{3}$}

The Philippines was the first ASEAN country to formulate a biotechnology regulatory system and has been a model for producers of agricultural biotechnology crops [8]. Regulation of biotechnology in the country is enacted through various executive and administrative orders. Some of these policies and guidelines that regulate biotechnology in the country are presented below.

Executive Order No. 430: Constituting the National Committee on Biosafety of the Philippines (Corazon C. Aquino, 10/10/ 1990). Conscious of the great potentials of biotechnology in raising the quality of life among Filipinos, and the risks posed by genetically engineered organisms and its release into the environment, Executive Order 430 constituted the National Committee on Biosafety of the Philippines under the Department of Science and Technology. The members of the committee will be composed of the representatives from DA, DENR and $\mathrm{DOH}$. The NCBP is mandated primarily to identify and evaluate potential hazards involved in initiating genetic engineering experiments or the introduction of new species and genetically engineered organisms and recommend measures to minimize risks; formulate and review national policies and

\footnotetext{
${ }^{3}$ The Philippine Policy Guidelines in Modern Biotechnology may be accessed at www.bic.searca.org.
} 
guidelines on biosafety, such as the safe conduct of work on genetic engineering, pests and their genetic materials for the protection of public health, environment and personnel and supervise the implementation thereof; formulate and review national policies and guidelines in risk assessment of work biotechnology, and supervise the implementation thereof; among others.

Executive Order No. 247: Prescribing guidelines and establishing a regulatory framework for the prospecting of biological and genetic resources, their byproducts and derivatives for scientific and commercial purposes; and other purposes (Fidel Ramos, 5/18/1995). In view of protecting and conserving the environment, Pres. Fidel Ramos issued Executive Order No. 247, which constituted the Inter-agency Committee on Biological and Genetic Resources, composed of the Undersecretary of DENR as Chair and Undersecretary of DOST as co-chairperson. The members will include a permanent representative of the Department of Agriculture, DOH, National Museum, academe experts and NGO. The powers and functions of the committee as outlined in Section 7 include (a) process applications for Research Agreement and recommend for approval thereof to the Secretary of DENR, $\mathrm{DOH}, \mathrm{DA}$ or DOST depending on the nature and character of the prospecting activity; (b) ensure that the conditions for Research Agreements ${ }^{4}$ are strictly observed; (c) determine the list and amount of biological and genetic materials taken from the area and ensure that these are

${ }^{4}$ E.O. No. 247 distinguished between an Academic Research Agreement and a Commercial Research Agreement. It specifies that if the research and collection of biological and genetic resources is intended, directly or indirectly, for commercial purposes, the agreement must be a Commercial Research Agreement. However, if the prospecting of the same materials is intended primarily for academic purpose, the agreement shall be Academic Research Agreement. complied with; (d) deputize and train appropriate agencies so as to ensure that no biological and genetic materials are taken from the Philippines and exported abroad, except under a valid Research Agreement. It shall also be ensured that the specimens collected have been deposited in the Philippines; (e) ensure that the rights of the indigenous and local communities where the collection or the researches are being conducted are protected, including the verification that the consent requirements in Section 3 and Section 4 are complied with.

Executive Order No. 514: Establishing the National Biosafety Framework, Prescribing Guidelines for its Implementation, Strengthening the National Committee on Biosafety of the Philippines, and Other Purposes (Gloria Macapagal Arroyo, 3/17/2006). This E.O. mandates the adoption of the National Biosafety Framework for the Philippines transition from NCBP which was constituted in 1990 to National Biosafety Framework (NBF) for the Philippines. One of the reasons that prompted the reorganization of the NCBP was the compliance with the administrative requirements of the Cartagena Protocol on Biosafety ${ }^{5}$. Section 2 enumerates the 12 principles based on national and international law that will govern the implementation of this Executive Order. These principles include the 1) Policy on Modern Biotechnology; 2) Policy on Sustainable Development; 3) A Balanced Approach; 4) Scientific Approach; 5) Socioeconomic, Cultural and Ethical Considerations, 6) Using precaution, 7) Transparency and

\footnotetext{
${ }^{5}$ The objective of this Protocol is to contribute to ensuring an adequate level of protection in the field of the safe transfer, handling and use of living modified organisms resulting from modern biotechnology that may have adverse effects on the conservation and sustainable use of biological diversity, taking also into account risks to human health, and specifically focusing on trans boundary movements [9].
} 
Public Participation; 8) Consensus building; 9) Principle of Subsidiarity; 10) Availability of Remedies; 11) International Obligations and Cooperation; and, 12) Efficient Administration and Timely Decision Making.

As regards decision making processes Section 5 provides that biosafety decisions shall be made in accordance to 1) standard of precaution; 2) risk assessment; and 3) role of environmental Impact assessment; 4) socioeconomic, ethical, cultural and other considerations; 5) decisions under the Cartagena Protocol; and, 6) monitoring and enforcement.

DA Administrative Order No. 8 Series of 2002: Rules and Regulations on the Importation and release into the Environment of Plants and Plant Products Derived from the Use of Modern Biotechnology (Gloria Macapagal Arroyo, 4//3/2002). As stated in Section 2 of this Administrative Order, the coverage includes the importation or release into the environment of:

1) Any plant which has been altered or produced through the use of modern biotechnology if the donor organism, host organism or vector or vector agent belongs to any of the genera or taxa classified by BPI as meeting the definition of plant pest or is a medium for the introduction of noxious weeds of; or

2) Any plant of plant product altered or produced through the use of modern biotechnology; which may pose significant risks to human health and the environment based on available scientific and technical information.

The Order specifies the approval processes of activities related to the importation of regulated articles ${ }^{6}$ for contained use ${ }^{7}$, field testing of regulated articles, propagation of regulated articles for direct use as food or feed, or for processing, and the ground and requirement for delisting of regulated article.

Any new technology poses risks and potential harms to users. In Section 3, DA Administrative Order No. 8 S.2002 enumerates the following risk assessment principles:

1. The risk assessment shall be carried out in a scientifically sound and transparent manner based on available scientific and technical information;

2. Lack of knowledge or scientific consensus shall not be interpreted as indicating a particular level of risk, an absence of risk, or an acceptable risk;

3. The identified characteristics of a regulated article and its use which have the potential to pose significant risks to human health and the environment shall be compared to those presented by the non-modified organism from which it is derived and use under the same condition;

4. Risk assessment shall be carried out cases by case and on the basis of transformation event; and,

5. If new information on the regulated article and its effects on human health and environment become available, the risk assessment shall be readdressed to determine whether the risk has changed or whether there is a need to amend the risk management strategies accordingly.

${ }^{6}$ It means any of the organisms listed in Section 2A, but excludes those that are delisted in accordance with section 14. See Section 1, Definition of Terms under DAAdministrative Order No. 8s, 2002.

${ }^{7}$ The term applies to the use of regulated article for research and development inside a physical containment facility intended to limit its contact with, and to provide for a high level of safety for, the general population and the environment and which has been inspected and approved by NCBP. See Section 1, Definition of Terms under DAAdministrative Order No. 8s, 2002. 


\section{Principles for the Oversight and Assessment of Nanotechnologies AND NaNomaterials}

In recent years, nanotechnology's tremendous potentials have been recognized worldwide. Like any new technology, nanotechnology poses not only great promises but also perils. In adopting nanotechnology in the Philippines, we can make use of the foundational principles for the oversight and assessment of nanotechnologies and nanomaterials, which was the product of the first Nanotechnology Strategy Summit in Washington, DC in January 2007 [10]. The principles that were enumerated are the following: 1) A Precautionary Foundation; 2) Mandatory Nano-specific Regulations; 3) Health and Safety of the Public and Workers; 4) Environmental Protection; 5) Transparency; 6) Public Participation; 7) Inclusion of Broader Impacts; and, (8) Manufacturer Liability.

The Precautionary Principle is formulated as follows "when an activity raises threats of harm to human health or the environment, precautionary measures should be taken even if some cause relationships are not fully established scientifically [11]. Andorno [12] claims that it has become an emergent principle of international law. Mandatory nano-specific regulations should be established on account of the novel properties of nanomaterials. The kind of regulation should be mandatory or by means of government oversight, and not through voluntary initiatives. There should also be protective measures to ensure the health and safety of the public and the workers in nanotechnology factories and workplaces. Environmental sustainability should be the goal that will balance the equation between material progress and environmental protection and preservation. However, due to knowledge gaps, the deleterious impacts of nanomaterials on health and the environment cannot be fully determined. Transparency should be observed.
The public has to be informed in order to make intelligent choices. Transparency requires labeling of products containing nanomaterials. Further, "the potential of nanotechnologies to transform the global social, economic, and political landscape makes it essential that the public fully participate in the deliberative and decision-making process" [10]. Furthermore, the broad range of complex issues related to nanotechnologies should be given focus. These will include its effects on the economies of developing countries; patent issues involved privatizing the building blocks of natural world, human enhancements, among others. These confounding issues necessitate that research on the ethical and societal impacts on nanotechnologies should be given adequate and substantial public funding. Lastly, manufacturer liability shifts the burden of proof to industry, making sure that marketable products are safe to use.

E.O. No. 514 contains specific provisions, to wit:

5.1 Standard of Precaution. In accordance with Article 10 (Par. 6) and Article 11 (Par. 8) of the Cartagena Protocol on Biosafety, lack of scientific certainty or consensus due to insufficient relevant scientific information and knowledge regarding the extent of the potential adverse effects of a genetically modified organism on the environment, particularly on the conservation and sustainable use of biological diversity, and on human health, shall not prevent concerned government departments and agencies from taking the appropriate decision to avoid or minimize such potential adverse effects. In such cases, concerned government department and agencies shall take the necessary action to protect public interest and welfare.

5.2 Risk Assessment. Risk assessment (RA) shall be mandatory and central in making biosafety decisions. It shall identify and 
evaluate the risks to human health and the environment, and if applicable, to animal health.

\subsection{Role of Environmental Impact} Assessment. The Application of EIA System to biosafety shall be determined by concerned departments and agencies subject to the requirements of the law and the standards set by the NCBP.

\subsection{Socioeconomic, Ethical, Cultural and} Other Considerations. Consistent with Article 26 of the Cartagena Protocol, concerned government departments and agencies may take into account socioeconomic considerations arising from the impact of regulated articles on the conservation and sustainable use of biological diversity, especially with regard to the value of biological diversity to indigenous and local communities.

Section 6. Access to Information. The right of the public and the relevant stakeholders to information related to biosafety decisions is recognized and shall always be respected in accordance with guidelines to be issued by the NCBP.

Section 7. Public Participation. The concerned government departments and agencies, in developing and adopting biosafety policies, guidelines and measures and in making biosafety decisions, shall promote, facilitate, and conduct public awareness, education, and meaningful, responsible, and accountable participation.

A closer examination of E.O. 514, S.2006 and the Principles of Oversight and Assessment of Nanotechnologies and Nanomaterials would reveal that they share common principles. These principles include standard of precaution, health and safety, environmental protection, transparency, public engagement and the inclusion of broader impacts. However, at present the country does not have nano-specific regulation and provisions for manufacturer laibility. At a glance, the Table below presents the comparative data.

\section{BioteCHNOLOGY AS AN EYE-OPENER: \\ SOME LESSONS FOR NANOTECHNOLOGY}

The case study of Richmond indicated that "though among the first nations to enact biosafety regulations, the Philippines' existing biosafety regulations do not adequately protect the environment because they lack enforcement power and leave gaps in coverage" [13]. The author also pointed out the need for a single regulatory body or a centralized authority with enforcement authority to address biosafety

\section{Comparison between the Principles for the Oversight and Assessment and Nanotechnologies and E.O. 514 S.2006}

\begin{tabular}{|c|c|}
\hline $\begin{array}{c}\text { Principles for the } \\
\text { Oversight and } \\
\text { Assessment of } \\
\text { Nanotechnologies and } \\
\text { Nanomaterials [10] }\end{array}$ & $\begin{array}{l}\text { Modern Biotechnology } \\
\text { Guidelines that were } \\
\text { adopted in the } \\
\text { Philippines }\end{array}$ \\
\hline $\begin{array}{l}\text { Precautionary } \\
\text { Foundation }\end{array}$ & $\begin{array}{c}\text { E.O. No. } 514 \\
\text { Section } 5.1 \\
\text { (Standard of Precaution) } \\
\end{array}$ \\
\hline $\begin{array}{l}\text { Mandatory Specific } \\
\text { Nano-regulation }\end{array}$ & No data available \\
\hline $\begin{array}{l}\text { Health and Safety of the } \\
\text { Public and Workers }\end{array}$ & $\begin{array}{c}\text { E.O. No. } 514 \\
\text { Section } 5.2 \\
\text { (Risk Assessment) } \\
\end{array}$ \\
\hline $\begin{array}{l}\text { Environmental } \\
\text { Protection }\end{array}$ & $\begin{array}{c}\text { E.O. No. } 514 \\
\text { Section } 5.3 \\
\text { (Environmental Impact } \\
\text { Assessment) }\end{array}$ \\
\hline Transparency & $\begin{array}{c}\text { E.O. No. } 514 \\
\text { Section } 6 \\
\text { (Access to Information) } \\
\end{array}$ \\
\hline Public Participation & $\begin{array}{c}\text { E.O. No. } 514 \\
\text { Section } 7.2 \\
\text { (Public Participation) } \\
\end{array}$ \\
\hline $\begin{array}{l}\text { Inclusion of Broader } \\
\text { Impacts }\end{array}$ & $\begin{array}{c}\text { E.O. No. } 514 \\
\text { Section } 5.4 \\
\text { (Socioeconomic, Ethical, } \\
\text { Cultural and other } \\
\text { considerations) } \\
\end{array}$ \\
\hline Manufacturer Liability & No data available \\
\hline
\end{tabular}


concerns and encourage strict penalties than mere revocation of permits or licenses for offenders.

Meanwhile, Greenpeace, an international NGO, raises doubts regarding the Philippine government's seriousness in addressing GMO risks. They claim that the budget allocated for risk assessment is small and has been shrinking and there is conflict of interest among the members of the regulatory bodies [14].

Biotechnology development generated strings of controversy particularly those surrounding GMOs and GM foods. Issues of safety, choice versus autonomy, confidence in the regulatory systems were raised and elicited negative reactions from the public. With nanotechnology, these issues will be revived. To prevent another backlash lessons have to be learned. With regard to new and emerging technologies, we need to employ proactive ethics pari passu reactive ethics. Proactive ethics anticipate problems and make predictions while reactive ethics respond to problems as they arise. In recent years, the European Union developed a framework on Responsible Research and Innovation. Responsible Reseach and Innovation means that societal actors work together during the whole research and innovation process and its outcomes, with the values, needs and expectations of European Society [15]. This framework may be adopted in the country and adapted to the local context.

\section{Conclusion}

In sum, the following points may be deduced from the foregoing discussions:

1) Reviewing the biotechnology guidelines in the Philippines, we may infer that there are existing standards to address risk and safety issues in biotechnology. First, E.O. No. 430 constituted the National Committee on Biosafety of the Philippines (NCBP), an interagency unit, whose primary mandate is to study the potential risks of genetically engineered organisms and to recommend measures to mitigate the risks Likewise, the guidelines were also set by E.O. No. 247 give clear guidelines for prospecting biological and genetic materials for research or commercial purposes. It protects Philippine biodiversity and safeguards the rights of indigenous people and local communities where researches are conducted. E.O. No. 514 establishes the Biosafety Framework of the Philippines based on 12 principles which were based on national and international laws. In particular, the NCBP was reorganized to be compliant with the Cartagena Protocol on Biosafety. DA Administrative Order No. 8 S.2002 addresses risk issues and enumerated the principles with which to assess risks with respect to the release of genetically engineered plant and plant products into the environment.

2) The Table shows that Modern Biotechnology Guidelines generally agrees with the Principles for the Oversight and Assessment of Nanotechnologies and Nanomaterials. The various executive and administrative orders explain that the Philippines clearly recognizes the role of new technologies in national development yet at the same time advocates its prudent use.

3) Nanotechnology is an emerging technology that has great potentials in improving people's lives. Like biotechnology, it broaches environmental, health and safety concerns. Furthermore, there are emerging ethical and societal concerns that need to be addressed. While many considered nanotechnology as extensions of ethical debates in biotechnology, there remains to be two essential issues that are nano-specific.

4) While the relevant E.O.s that we have cited proved its conformity with most of the principles for oversight of nanotechnologies 
and nanomaterials (precautionary foundation, health and safety of the public and workers, environmental protection, transparency, public participation, inclusion of broader impacts and manufacturer liability), we have yet to address two additional requirements that are specified in the Principles of Oversight and Assessment of Nanotechnologies and Nanomaterials, namely, mandatory nano-specific regulations and manufacturer liability. Following the U.S. model, the Philippine laws subsume nanoregulation under the scope of existing regulatory schemes.

Due to the novel properties of nanomaterials and the existing knowledge gaps in nanotechnology, nanoregulation would be a big challenge. The report suggests that regulation should be mandatory and not merely voluntary since these "initiatives often delay or weaken essential regulation, forestall public involvement, and limit public access to vital environmental safety and health data" [10]. The report favors the European Union's adoption of a more precautionary stance towards regulation of nanotechnologies. The burden rests on industry to prove that there is no risk in products containing nanomaterials. All those who market nano-products are held accountable for any liability that will arise from their products. The report mentions that other forms liability includes "negligence, derivative liability, nuisance, fraud and misrepresentation" [10].

5) While there are mechanisms for regulating biotechnology that are in place, which may work well with nanotechnology development in the country, they are not sufficient to address a maelstrom of issues and concerns. Clearly, there is a big room for the improvement of regulatory standards. Temporary fixes in the gaps are inadequate measures. Laws should be enacted by duly constituted authorities and stricter penalties should be imposed against offenders.
6) While we may agree with the common observation that as the science of nanotechnology leaps ahead, the ethics lags behind; the situation, however, is not irredeemable. Prudence counsels us to learn from our experiences with past technologies. Just as it is profitable to fix our gaze and pin our hopes on nanotechnology, it is equally beneficial to look back at the lessons of biotechnology before we make the leap.

\section{REFERENCES}

[1] Hansson SO. Great Uncertainty About Small Things. Techne. 2004; 8:26-35.

[2] Bennett-Woods D. Nanotechnology, Ethics and Society (New York: CRC Press, 2008).

[3] Clift R. Nanotechnology: An Example of Risk Management and Regulation in an Emerging Technology (Special Session of the Joint Meeting: Potential Implications of Manufactured Nanometerials for Human Health and Environmental Safety; 2005 June 7.

[4] Meili C. Nanoregulation A multi-stakeholderdialogue-approach towards a sustainable regulatory framework for nanotechnologies and nanosciences. (St. Gallen: The Innovation Society, 2006).

[5] Matsuura JH. Nanotechnology Regulation and Policy Worldwide. (Massachusetts: Artech House, 2006).

[6] Mantovani E, Porcari A, Morrison MJ \& Geertsma RE. Developments in Nanotechnologies Regulations and Standards 2010 (Report of the Observatory Nano, 2010 June). www.observatorynano.eu

[7] Dayrit FM. Nanotechnology Prospects and Priorities. (Taguig City: DOST-PCIEERD, 2012).

[8] Hautea RA \& Escaler M. Plant biotechnology in Asia. AgBioForum 2004; 7:2-8.

[9] Secretariat of the Convention on Biological Diversity. Cartagena Protocol on Biosafety to the Convention on Biological Diversity: text and annexes. (Montreal: Secretariat of the Convention on Biological Diversity, 2000).

[10] International Center for Technology Assessment. Principles of the Oversight of Nanotechnologies and Nanomaterials. (San Francisco: International Center for Technology Assessment, 2007).

[11] Wingspread Statement on the Precautionary Principle, 1998. 
[12] Andorno R. The Precautionary Principle: A New Legal standard for a Technological Age. J. Int. Biotechnol. Law 2004; 1:11-19.

[13] Richmond CL. Genetically modified crops in the Philippines: Can existing biosafety regulations adequately protect the environment? Pac. Rim L. \& Poly. J. 2006; 15:569-598.
[14] Greenpeace. Ties that bind: Regulatory recapture in the country's GMO approval process (2007 October 2). http:// www.greenpeace.org

[15] European Union Responsible Research and Innovation. Europe's ability to respond to societal challenges 2012. https://ec.europa.eu 